\title{
Psychological interventions using virtual reality for pain associated with medical procedures: A systematic review and meta-analysis
}

\author{
Raluca Georgescu ${ }^{\mathrm{a}, \mathrm{b}}$, Liviu A. Fodor ${ }^{\mathrm{a}, \mathrm{b}}$, Anca Dobrean ${ }^{\mathrm{a}, \mathrm{c}}$, Ioana A. Cristea ${ }^{\mathrm{c}, \mathrm{d}, *}$
}

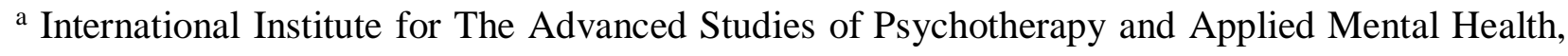
Babeș-Bolyai University, Cluj-Napoca, Romania

b Evidence Based Psychological Assessment and Interventions Doctoral School, Babeș-Bolyai University, Cluj-Napoca, Romania

c Department of Clinical Psychology and Psychotherapy, Babeş-Bolyai University, Republicii Street 37, 400015, Cluj-Napoca, Romania

${ }^{\mathrm{d}}$ Department of Clinical, Neuro and Developmental Psychology Vrije Universiteit Amsterdam, The Netherlands

* Corresponding author at: Department of Clinical Psychology and Psychotherapy, Babeş-Bolyai University, Republicii Street 37, 400015 Cluj-Napoca, Romania

E-mail address: ioana.cristea@ubbcluj.ro (Cristea, I.A.)

Wordcount: 4804

This is a post-print (accepted version without publisher corrections) of the article published in Psychological Medicine DOI: https://doi.org/10.1017/S0033291719001855 


\begin{abstract}
Background: Virtual reality (VR) may enhance the effectiveness of psychological interventions for acute pain. We conducted a meta-analysis to assess the efficacy and safety of VR-based interventions for pain associated with medical procedures.
\end{abstract}

\title{
Methods:
}

We searched PubMed, EMBASE, the Cochrane Library, and PsycINFO until June $17^{\text {th }}, 2018$. We identified 36 randomized controlled trials (RCTs), comparing VR-based psychological interventions to usual care, for pain intensity (primary outcome) or affective and cognitive components of pain (secondary outcomes), assessed real-time or retrospectively. Two independent reviewers performed study selection and data extraction. Risk of bias was independently evaluated by three raters using the revised Cochrane Collaboration tool. A randomeffects model using the Paule and Mandel estimator was used for pooling effect sizes.

Results: 27 RCTs (1452 patients) provided enough data for meta-analysis. Compared to usual care, VR-based interventions reduced pain intensity both real-time ( 9 RCTs, Hedges' $g=0.95$, 95\% CI 0.32 to 1.57 ) and retrospectively (22 RCTs, $g=0.87,95 \%$ CI 0.54 to 1.21 ). Results were similar for cognitive ( 8 RCTs, $g=0.82,95 \%$ CI 0.39 to 1.26 ) and affective pain components (14 RCTs, $g=0.55,95 \%$ CI 0.34 to 0.77 ). There was marked heterogeneity, which remained similar in sensitivity analyses. Across domains, few trials were rated as low risk of bias and there was evidence of publication bias. Adverse events were rare.

Conclusions: Though VR-based interventions reduced pain for patients undergoing medical procedures, inferring clinical effectiveness is precluded by the predominance of small trials with substantial risk of bias and by incomplete reporting. 
Acute pain is an often unavoidable side-effect of medical procedures, such as treatment for burns (Tsirigotou 1993; Norman \& Judkins 2004), cancer (van den Beuken-van Everdingen et al. 2016), or dental (Pak \& White 2011; da Costa et al. 2012); surgery (Sommer et al. 2008), or intensive care procedures (Barr et al. 2013). Inappropriate management of acute pain is accompanied by protracted hospitalization (Chan et al. 2013), short-and long-term costs (Torrati et al. 2000) and represents a risk factor for developing chronic or persistent pain (Turk \& Okifuji 2002; Breivik et al. 2013).

Psychological interventions for acute pain management, such as relaxation (Seers \& Carroll 1998) or distraction (Kleiber \& Harper 1999) attempt to disrupt the process of allocating attentional resources to pain. However, not all patients are equally capable of making use of these techniques and effectively regulating their attention (Seers \& Carroll 1998), particularly in situations of increased pain salience and in the absence of goal directed motivation (Verhoeven et al. 2010). Though psychological interventions are generally effective in adults, particularly in some contexts like burn wound care (Scheffler et al. 2017), their effectiveness is more limited in children and adolescents (Uman et al. 2008), who are particularly ill-equipped to modulate pain attention, probably due to lower inhibition and working memory abilities (Verhoeven et al. 2014). Virtual reality (VR) technology is a promising development for enhancing the effectiveness of traditional interventions, such as distraction or relaxation, for acute pain. An immersive (Brooks 1999) and multi-sensorial experience (Gallace et al. 2012), achieved through a combination of technologies (i.e., head-mounted displays, vibro-tactile gloves, individualized sounds, and gesture-sensing joysticks), along with the possibility of active exploration could facilitate the shift of attention away from the painful stimuli or the experience of pain, aiding effective distraction and reshaped pain perception (Gold et al. 2007; Piskorz \& Czub 2014). The 
technology could be effectively exported in medical care settings (Li et al. 2017) as a potentially cost-effective tool (Malloy \& Milling 2010), particularly since recent user-friendly developments (e.g., smaller headsets, intuitive controllers) do not require special training and could easily be used by medical providers (e.g., nurses). Moreover, some newer VR technologies like the multimodal device (MMD) are especially designed for medical settings with younger patients. The technology uses a hand-held screen as an alternative to HMD or video glasses to protect visual functions of children under 7 years of age and programs include tailored stories and interactive games that prepare children for undergoing medical procedures or are used for distraction during painful ones.

Single trials of VR-based interventions for acute pain are accruing, with both encouraging (Schmitt et al. 2011; Gold \& Mahrer 2018) and mixed results (Wint et al. 2002; Walker et al. 2014). One meta-analysis (Scheffler et al. 2017) of non-pharmacological treatments in general for adults undergoing burn care reported large effects for distraction interventions, particularly when these used VR, but the number of studies in this subgroup was small and outcomes of pain intensity, affective and cognitive components were combined. Another meta-analysis (Chan et al. 2018) examined VR-based treatments for painful clinical interventions and reported a moderate ES of 0.49 for maximum self-rated pain. Yet several clinically and theoretically important aspects were not investigated. Trials often also include additional measures of pain intensity (e.g., pain threshold), as well other pain-related outcomes (e.g., distress) and involve other assessors beside self-report. Moreover, the timepoint of pain assessment is subject to a clinically important distinction, between real-time assessments "during", and retrospective evaluations "after ", medical procedures. Comparisons between VR and other active treatments were not examined, though these could indicate whether observed effects are specific to VR or rather attributable to 
non-specific factors like novelty. Several potential moderators of clinical or theoretical importance were not examined. VR-enhanced interventions might be particularly effective for young participants, less able to engage in standard distraction. An important theoretical question is whether VR enhances the effectiveness of regular distraction. Possible moderating effects could result from concomitant analgesic use or the type of VR system employed. Finally, possible publication bias, as well potential adverse effects were not previously reported.

Hence, our goal was to assess the efficacy and safety of VR-based psychological interventions for pain associated to medical procedures, expanding on the issues identified above.

\section{Method}

\section{Data Sources and Searches}

We searched the National Library of Medicine via PubMed, Embase, PsycInfo and Cochrane Library databases in June 2018, using the following keywords: "virtual reality", "game”, “interface”, “immersion”, "virtual reality exposure therapy”, “pain”, "burn”, “wound”, and "injuries" (complete search strings in Appendix 1). We also searched the references of narrative and systematic previous reviews.

\section{Study Selection}

Eligible studies were: (1) randomized controlled trials (RCTs), in (2) patients of any age undergoing a painful procedure delivered in a medical setting comparing (3) a VR-based psychological intervention (4) with treatment as usual, (e.g., analgesics alone, standard distraction) or an active comparator devised by investigators (psychological, pharmacological), (5) reporting any pain outcomes, (6) published in peer-reviewed journals. VR interventions could be stand-alone or combined with another intervention (e.g. pain medication), provided the same 
ancillary intervention was also administered to the control group. Both crossover and parallel designs were eligible. No language restrictions were used.

We excluded the dissertations and conferences abstracts. For multiple reports of the same trial, we used the most complete one. One researcher screened all abstracts and flagged potentially eligible studies (RG). These were then retrieved full-text and independently assessed by two independent researchers (RG, LF). Disagreements were resolved by discussion with a third author (IC).

\section{Data extraction}

The primary outcome of interest was pain intensity (i.e., mean pain intensity, pain threshold and worst pain), measured by Visual Analogue Scale (VAS) or other clinical rating scales (e.g. Graphical Rating Scale/GRS, Faces Pain Scale), assessed real-time (i.e., during the medical procedure) or retrospectively (i.e., after the procedure). For outcomes assessed by more observers (e.g., child, parent, nurse), we extracted data for each. Secondary outcomes were cognitive (e.g., time spent thinking about pain and worry), and affective components of pain (e.g., pain unpleasantness, anxiety and distress) as assessed by VAS, GRS or other clinical rating scales (e.g., Symptom Distress Scale; Face, Leg, Activity, Cry, Consolability Scale).

For each included trial, we extracted information about: (a) study design (i.e., parallel, crossover); (b) medical procedure (e.g., dressing change, physical therapy); (c) condition requiring medical procedure (e.g., burn, cancer, dental treatment); (d) age group (i.e., children, adults or mixed); (e) recruitment (i.e., clinical, community); (f) VR-based intervention (e.g., distraction, psycho-education); (g) TAU or active comparator condition; (h) numbers of patients randomized in the treatment groups; (i) number of sessions; (j) concomitant analgesic use, and, if present the class of drugs (e.g., opioids, nonsteroidal anti-inflammatory drugs (NSAIDs), local); 
(k) VR system (e.g., head-mounted display/HMD, video glasses) and the number of interactive components (e.g., visual feedback, sound, navigation); (l) assessment of presence and immersion, if present; (m) adverse effects associated with VR; (n) number of drop-outs in the treatment groups; (p) VR program developer trial investigator (yes/no); (o) country of provenience. One reviewer $(\mathrm{RG})$ extracted descriptive and outcome data. Another reviewer (IC) independently checked one third of the trials.

\section{Risk of bias (Rob) assessment}

RoB was assessed with the revised Cochrane Collaboration tool (Higgins et al. 2016), separately for parallel and crossover designs, using templates with incorporated decision algorithms (available at: http://www.riskofbias.info/welcome/rob-2-0-tool ). We evaluated sources of bias in five domains: (a) the adequate generation of the allocation sequence, (b) deviations from intended interventions (including blinding of participants and research personnel), (c) handling of incomplete outcome data, (d) measurement of the outcome (blinding of outcome assessors) and (d) selection of the reported results. For deviations from intended interventions, we rated studies as low risk of bias if the investigators described any valid method of blinding participants or research personnel, or if they specifically mentioned attempting to control for possible deviations. We rated studies as low risk for missing outcome data if all randomized participants were included in the analysis, the authors specified there were no or less than 5\% missing data or intent-to-treat analyses were used, (Higgins et al. 2016). For blinding of assessors, self-report assessments were considered high risk (Higgins et al. 2016). In crossover trials, we also evaluated potential carry-over effects, by weighing whether the time elapsed between the successive interventions was sufficient to prevent carry-over. For selective outcome reporting, we extracted trial registration numbers in the paper and, if available, published 
protocols or other secondary reports. We also computed an overall RoB score for each study by awarding 1 point for each bias source rated as low risk, for use in subsequent sensitivity analyses. Ratings were independently done by three researchers (RG, LF, and IC) and disagreements were discussed and resolved.

\section{Data Synthesis and Analysis}

\section{Meta-analyses}

We used the software packages Comprehensive Meta-Analysis (CMA v. 2.2.064) for computing study-level effect estimates and Stata SE 14.0 (STATA Corp., Inc., College Station, TX) packages Admetan (Fisher 2015, 2019) for pooling, Metabias (Harbord et al. 2009) for testing small study effects and Confunnel (Palmer et al. 2008) for visualization. Effect sizes (ESs) were calculated as standardized mean difference (SMD) for each comparison, transformed in the adjusted Hedges' $g$ (Hedges \& Olkin 1985) to correct for the small sample size of most studies. In parallel designs, SMDs represent the difference between the means of the VR and the control group at each timepoint (real-time, retrospective), divided by the pooled standard deviations of the two groups, with positive values indicating superiority of VR-based interventions. When means and standard deviations were not reported, we computed the SMD from alternative statistics (Borenstein et al. 2009), such as $t$ values or $p$ values from independent group comparisons at the time-point of interest, and sample sizes.

In crossover designs, we primarily relied on individual participant means in each period and derived SMDs by computing within-participant mean differences, corresponding standard errors (SEs) for the differences, and the correlation between intervention and control (Elbourne et al. 2002). When individual participants means were not available, we computed the SMD from 
the within-subject mean differences, SD of differences and sample size, paired-sample $t$ values or p values (Elbourne et al. 2002; Borenstein et al. 2009).

If no usable information was available, authors were contacted. If a trial employed more comparisons arms from the same category, only data for one of the eligible arms was used (i.e., the most similar to the other included trials was used). In the case of multiple VR intervention groups, we computed and averaged separate ESs for each comparison with a control group. If an outcome (e.g., pain intensity) was assessed by more observers (e.g., self-report, others), we computed ESs both separately and across all assessors. To facilitate the clinical interpretation, we also report absolute benefits as numbers-needed-to-be treated (NNT), the number of patients that have to be treated in order to generate one additional positive outcome (Laupacis et al. 1988), computed with the Kraemer and Kupfer formula (Kraemer \& Kupfer 2006).

We aggregated individual ESs separately for: pain intensity as sensory component of pain measured real-time and, respectively, retrospectively; time spent thinking about pain and worry as cognitive components of pain; and pain unpleasantness, anxiety, distress as affective components of pain. Comparisons against TAU or other active competitors were aggregated separately.

We pooled studies with a random-effect model. Based on previous systematic reviews and the particularities of the population and setting, we expected most studies to use small samples. Therefore, we used the Paule and Mandel estimator (Paule \& Mandel 1982) for between-study variance $\left(\tau^{2}\right)$, as recommended by a recent review of estimation methods (Veroniki et al. 2016). We also applied the Hartung-Knapp-Sidik-Jonkman (HKSJ) variance correction (Hartung \& Knapp 2001; Sidik \& Jonkman 2002), with truncation of correction factor at 1, recommended for random-effects meta-analysis with few studies (Röver et al. 2015). We evaluated statistical heterogeneity with the $I^{2}$ statistic, which shows the percentage of total variation across studies due 
to heterogeneity. Values across $25 \%, 50 \%$ and $75 \%$ suggest low, moderate and high heterogeneity (Higgins et al. 2003). We used the Q-profile (QP) method for constructing the confidence intervals around heterogeneity estimates, shown to be adequate in terms of coverage probabilities even in small samples (Viechtbauer 2007). We also report predictive intervals (PI) (Higgins et al. 2009), as the confidence interval of the approximate predictive distribution of future trial, considering heterogeneity.

\section{Sensitivity and subgroup analyses}

As we expected high heterogeneity and few studies with low RoB across domains, we computed two additional meta-analysis models: the Henmi-Copas approximate exact distribution, which produces a confidence interval for the pooled effect robust to publication bias (Henmi \& Copas 2010), and the Quality Effects model, which integrates study quality into pooled estimate, favoring (i.e., assigns larger weights) both larger and better trials (Doi et al. 2015). We used the overall RoB score as a proxy for study quality.

We also conducted sensitivity analyses: i) excluding outliers (no overlap between the $95 \%$ CIs of the pooled ES with those of single trials); ii) excluding trials using the new technology MMD; iii) excluding comparisons where the control group received no treatment; iv) for burns dressing change; v) for children participants; vi) separately for outcome assessors (self-report, other-reports); vii) separately by design (parallel, crossover); viii) for trials with at least 20 participants randomized/arm; ix) excluding trials characterized by their authors as pilot or feasibility studies.

We had initially planned several of the sensitivity analysis as subgroup analysis, but realized the number of studies was too small. Using a rule of at least 10 studies per characteristic modelled (Higgins \& Green 2011, sec. 9.6.5.1) and expecting at least one comparison with over 
20 studies, we retained two characteristics: one of practical relevance- use concomitant analgesic use (present versus absent), and one of theoretical relevance- use of standard distraction in the control group, in the case this group received an intervention $(\mathrm{Y} / \mathrm{N})$. The correlation between the two characteristics was computed (Cramér's V for nominal data) to reduce the risk of confounding (Higgins \& Green 2011, sec. 9.6.5.6) . The statistical significance threshold was set at 0.025, Bonferroni corrected for multiple comparisons.

\section{Small study effects and publication bias}

We visually examined funnel plot asymmetry and constructed contour enhanced funnel plots (Peters et al. 2008), with contour lines indicating regions where the test of treatment effects was significant for various statistical significance levels. For comparisons with at least 10 ESs, we also conducted Eggers` test of the intercept (Egger et al. 1997). We also addressed publication bias in sensitivity analysis with the Henmi-Copas estimate.

\section{Results}

\section{Study selection (Figure 1, Appendix 1)}

We identified 3381 records and screened 1943 after removal of duplicates. We retrieved full-texts of 68 reports and further selected a total of 36 RCTs for inclusion. Figure 1 represents the flowchart of the inclusion process following the PRISMA guidelines (Moher et al. 2009). For 12 RCTs, data was insufficient for ES calculation and authors were contacted, with a second reminder if necessary. Data for ES calculation were retrieved in 3/12 cases (Bentsen et al. 2001; Maani et al. 2011; Schmitt et al. 2011). In total, 27 included trials had sufficient information for ES calculation, and were included in the meta-analysis. 


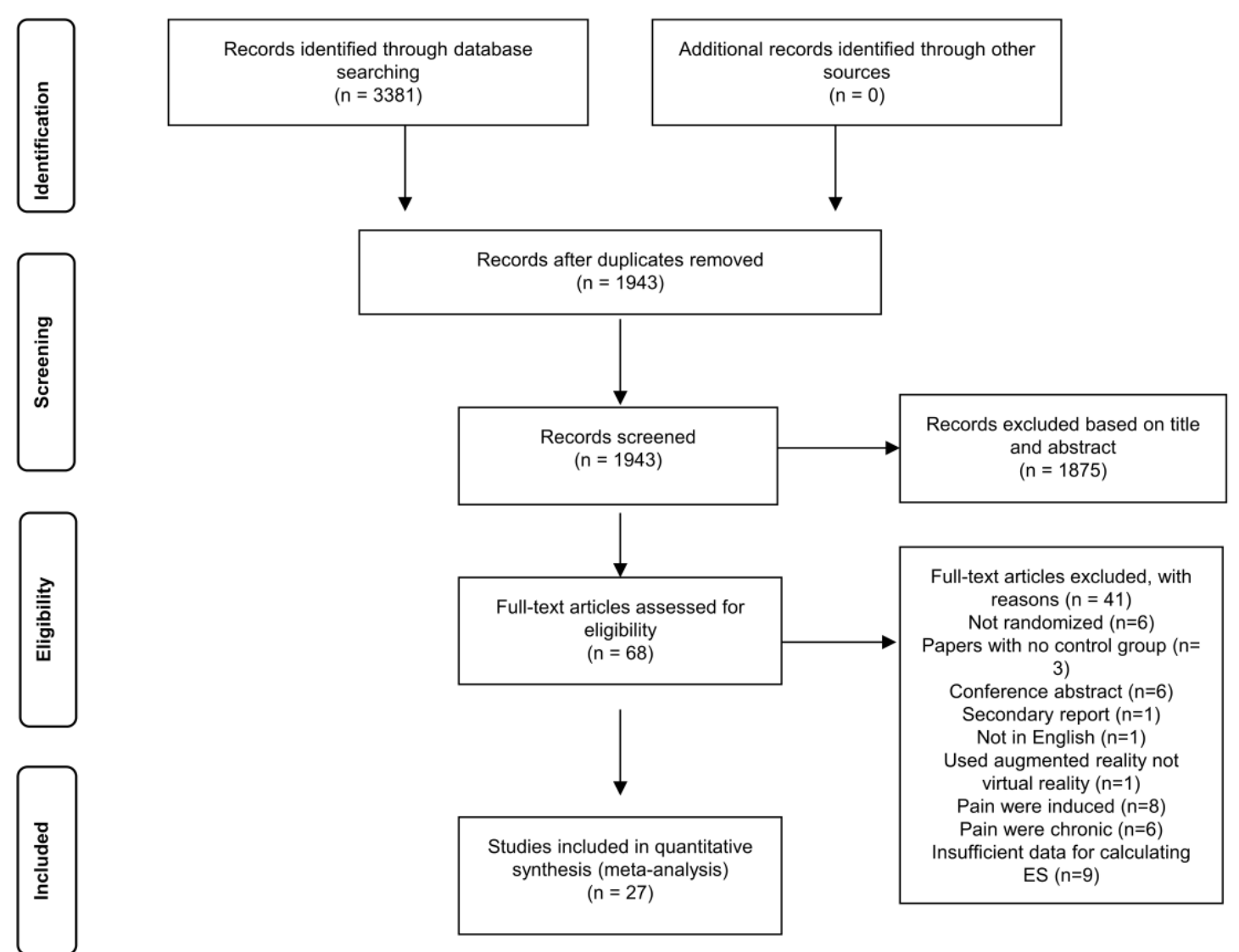

Figure 1. PRISMA flow-diagram of the study selection process

\section{Characteristics of included studies (Table 1, Table S1)}

1452 patients were treated (659 with VR-based interventions and 793 with TAU or another active intervention). The average number of randomized participants in the VR arm was 25 , and the average number of drop-outs was less than 1 . Five trials had 10 or less participants per arm. Fourteen trials had parallel design and thirteen had crossover design ( $\mathrm{AB} \mid \mathrm{B} A$ format). Most trials were focused on burns, for new or chronic wounds, either for dressing change (12), or physical therapy (5), with Total Burn Surface Area (TBSA) ranging from 1\% to $15 \%$. Five studies were conducted for pain and distress related with needle procedures (e.g. during intravenous (IV) port access placement or phlebotomy), two with dental treatment and another two with 
chemotherapy. Most participants were recruited from clinical settings (26). Thirteen studies targeted children and youth, ten, adults and four, mixed samples. All but one of the VR-based interventions used distraction. Twenty-seven trials had a TAU comparison: no treatment (5), analgesics alone (16), distraction (2), analgesics plus distraction (4). Additionally, four trials also included an active comparator arm, designed for the purpose of the trial (e.g., external cold and vibration group (Gerçeker et al. 2018) or video game group (Gershon et al. 2004). Interventions ranged from one to five sessions and were all conducted individually. In eighteen studies, all participants received concomitant analgesics, most frequently Oxycodone opioids. The most used VR system was HMD (15), followed by video glasses (8) and MMD (3). The VR developer was also an investigator in twelve trials. 
Table 1. Characteristics of included studies

\begin{tabular}{|c|c|c|c|c|c|c|c|c|c|c|c|c|c|}
\hline Study & Design $^{a}$ & Procedure $^{\mathrm{b}}$ & Cond $^{c}$ & Age gr $^{d}$ & Recr. $^{e}$ & $\begin{array}{l}\mathbf{N}_{\text {rand }} \\
\mathbf{V R}^{\mathbf{f}}\end{array}$ & $\begin{array}{l}\mathrm{N}_{\text {rand }} \\
\text { Co. }^{\mathrm{g}}\end{array}$ & Interv. ${ }^{\mathbf{h}}$ & Control $^{i}$ & $\begin{array}{c}\mathbf{N} \\
\text { sess }^{\mathbf{j}}\end{array}$ & $\begin{array}{c}\text { Pain outcome } \\
\text { assessed (Scale) }\end{array}$ & $\begin{array}{l}\text { Conc } \\
\text { analg }{ }^{1}\end{array}$ & $\underset{\mathbf{m}}{\operatorname{Prov}}$ \\
\hline Bentsen, 2001 & $\mathrm{CO}$ & $\begin{array}{l}\text { Restorative } \\
\text { Tx. }\end{array}$ & Dental Tx. & Child & Comm & 23 & 23 & VR-Distr. & No Tx. & 1 & $\begin{array}{c}\text { Pain (VAS) } \\
\text { Unpleas (VAS) }\end{array}$ & - & $\mathrm{Dk}$ \\
\hline Brown, 2014 & $\mathrm{P}$ & Dress Ch & $\begin{array}{l}\text { Burn } \\
\text { (TBSA } \\
>15 \%)\end{array}$ & Child & Clin & 47 & 52 & $\begin{array}{l}\text { VR-Distr. } \\
\text { + analg. }\end{array}$ & $\begin{array}{l}\text { Analg+ } \\
\text { Distr. }\end{array}$ & 2 & $\begin{array}{c}\text { Pain (FPS-R) } \\
\text { Distress (FLACC) }\end{array}$ & Opioids & $\mathrm{Au}$ \\
\hline $\begin{array}{l}\text { Carrougher, } \\
2009\end{array}$ & $\mathrm{CO}$ & $\begin{array}{l}\text { Physical } \\
\text { therapy }\end{array}$ & $\begin{array}{c}\text { Burn } \\
\text { (Level I) }\end{array}$ & Adults & Clin & 39 & 39 & $\begin{array}{l}\text { VR-Distr. } \\
\text { + analg. }\end{array}$ & Analg. & 1 & $\begin{array}{c}\text { Pain } \\
\text { Unpleas (VAS) } \\
\text { Time think (VAS) }\end{array}$ & Opioids & US \\
\hline Chan, 2007 & $\mathrm{CO}$ & Dress Ch & Burn & Child & Clin & 8 & 8 & VR-Distr. & Analg. & 1 & $\begin{array}{l}\text { Pain (WB- } \\
\text { FACES) }\end{array}$ & $\begin{array}{l}\text { Opioids + } \\
\text { NSAIDs }\end{array}$ & ES \\
\hline Frere, 2001 & $\mathrm{CO}$ & Dental Tx. & Dental Tx. & Adults & Clin & 25 & 25 & VR-Distr. & No Tx. & 1 & $\begin{array}{c}\text { Pain (GRS) } \\
\text { Anxiety (DFS) }\end{array}$ & - & US \\
\hline Gerceker, 2018 & $\mathrm{CO}$ & Phlebotomy & $\begin{array}{l}\text { Medical } \\
\text { analyses }\end{array}$ & Child & Clin & 40 & 40 & VR-Distr. & No Tx. & 1 & $\begin{array}{l}\text { Pain (WB- } \\
\text { FACES) }\end{array}$ & - & TR \\
\hline Gershon, 2004* & $\mathrm{P}$ & $\begin{array}{l}\text { Port access } \\
\text { placement }\end{array}$ & Cancer Tx. & Child & Clin & 22 & 15 & $\begin{array}{l}\text { VR-Distr. } \\
\text { + analg. }\end{array}$ & Analg. & 1 & $\begin{array}{c}\text { Pain (VAS) } \\
\text { Behav Obs } \\
\text { (CHEOPS) } \\
\text { Anxiety(VAS) }\end{array}$ & Local & US \\
\hline Gold, 2006 & $\mathrm{P}$ & IV Placement & MRI or CT & Child & Clin & 10 & 10 & VR-Distr. & Analg. & 1 & $\begin{array}{c}\text { Worry (WB- } \\
\text { FACES/ FACES - }\end{array}$ & Local & US \\
\hline
\end{tabular}


procedure

\begin{tabular}{|c|c|c|c|c|c|c|c|c|c|c|c|c|c|}
\hline & & & & & & & & & & & Pain (VAS) & & \\
\hline Gold, 2018 & $\mathrm{P}$ & Phlebotomy & $\begin{array}{l}\text { Medical } \\
\text { analyses }\end{array}$ & Child & Clin & 70 & 71 & VR-Distr. & Distr. & 1 & $\begin{array}{c}\text { Anxiety } \\
\text { (VAS/FAS) }\end{array}$ & - & US \\
\hline Guo, 2014 & $\mathrm{P}$ & Dress $\mathrm{Ch}$ & $\begin{array}{c}\text { Hand } \\
\text { wounds }\end{array}$ & Adults & Clin & 49 & 49 & VR-Distr. & No Tx. & 1 & Pain (VAS) & - & $\mathrm{CN}$ \\
\hline Hoffman, 2000 & $\mathrm{CO}$ & $\begin{array}{l}\text { Physical } \\
\text { therapy }\end{array}$ & Burn & Adults & Clin & 12 & 12 & $\begin{array}{l}\text { VR-Distr. } \\
\text { + analg. }\end{array}$ & Analg. & 1 & $\begin{array}{c}\text { Pain (VAS) } \\
\text { Time think (VAS) } \\
\text { Unpleas (VAS) }\end{array}$ & Opioids & US \\
\hline Hoffman, 2001 & $\mathrm{CO}$ & $\begin{array}{l}\text { Physical } \\
\text { therapy }\end{array}$ & $\begin{array}{c}\text { Burn } \\
\text { (severe } \\
\text { burn) }\end{array}$ & Mixed & Clin & 7 & 7 & $\begin{array}{l}\text { VR-Distr. } \\
+ \text { analg. }\end{array}$ & Analg. & 3 & $\begin{array}{c}\text { Pain (VAS) } \\
\text { Time think (VAS) } \\
\text { Unpleas (VAS) }\end{array}$ & NR & US \\
\hline Hoffman, 2008 & $\mathrm{CO}$ & Dress Ch & Burn & Mixed & Clin & 11 & 11 & $\begin{array}{l}\text { VR-Distr. } \\
+ \text { analg. }\end{array}$ & Analg. & 1 & $\begin{array}{c}\text { Pain (GRS) } \\
\text { Time think (GRS) } \\
\text { Unpleas (GRS) }\end{array}$ & Opioids & US \\
\hline Hua, 2015 & $\mathrm{P}$ & Dress Ch & Wounds & Child & Clin & 33 & 32 & VR-Distr. & Distr. & 1 & $\begin{array}{c}\text { Pain } \\
\text { (FACES/VAS) } \\
\text { Distress (FLACC) }\end{array}$ & - & $\mathrm{CN}$ \\
\hline $\begin{array}{l}\text { Jahanishoorab, } \\
2015\end{array}$ & $\mathrm{P}$ & $\begin{array}{c}\text { Episiotomy } \\
\text { Repair }\end{array}$ & Surgery & Adults & Clin & 15 & 15 & $\begin{array}{l}\text { VR-Distr. } \\
\text { + analg. }\end{array}$ & Analg. & 1 & Pain (NRP) & Local & IR \\
\hline Jeffs, 2014 & $\mathrm{P}$ & Dress Ch & $\begin{array}{l}\text { Burn } \\
\text { (FDC) }\end{array}$ & Child & Clin & 8 & 10 & $\begin{array}{l}\text { VR-Distr. } \\
\text { + analg. }\end{array}$ & Analg. & 1 & $\begin{array}{l}\text { Pain (APPT- } \\
\text { WGRS) }\end{array}$ & Opioids & US \\
\hline Kipping, 2012 & $\mathrm{P}$ & Dress Ch & $\begin{array}{c}\text { Burn } \\
\text { (TBSA> } \\
1 \%)\end{array}$ & Child & Clin & 20 & 21 & $\begin{array}{l}\text { VR-Distr. } \\
\text { + analg. }\end{array}$ & $\begin{array}{c}\text { Analg. + } \\
\text { distr. }\end{array}$ & 1 & $\begin{array}{c}\text { Pain (VAS) } \\
\text { Distress (FLACC) }\end{array}$ & $\begin{array}{c}\text { Opioids + } \\
\text { NSAIDs }\end{array}$ & $\mathrm{Au}$ \\
\hline
\end{tabular}




\begin{tabular}{|c|c|c|c|c|c|c|c|c|c|c|c|c|c|}
\hline $\begin{array}{c}\text { Konstantatos, } \\
2009\end{array}$ & $\mathrm{P}$ & Dress Ch & Burn & Adults & Clin & 43 & 43 & VR-Rlx. & Analg. & 1 & Pain (VAS) & Opioids & $\mathrm{Au}$ \\
\hline Maani, 2011 & $\mathrm{CO}$ & Dress Ch & Burn & Adults & Clin & 12 & 12 & $\begin{array}{l}\text { VR-Distr. } \\
\text { + analg. }\end{array}$ & Analg. & 1 & $\begin{array}{c}\text { Pain }(\text { GRS }) \\
\text { Time think }(\text { GRS })\end{array}$ & Opioids & US \\
\hline Miller, 2010 & $\mathrm{P}$ & Dress Ch & $\begin{array}{c}\text { Burn } \\
(\text { TBSA > } \\
1 \%)\end{array}$ & Child & Clin & 20 & 20 & $\begin{array}{l}\text { VR- } \\
\text { Psychoed } \\
\text { \& Distr. + } \\
\text { anlag. }\end{array}$ & $\begin{array}{c}\text { Analg. + } \\
\text { distr. }\end{array}$ & 3 & $\begin{array}{c}\text { Pain (WB- } \\
\text { FACES/VAS) } \\
\text { Distress (FLACC) }\end{array}$ & $\begin{array}{l}\text { Opioids + } \\
\text { NSAIDs }\end{array}$ & $\mathrm{Au}$ \\
\hline Miller, 2011 & $\mathrm{P}$ & Dress Ch & $\begin{array}{l}\text { Burn } \\
\text { (TBSA } \\
\geq 1 \%)\end{array}$ & Child & Clin & 20 & 20 & $\begin{array}{l}\text { VR-Distr. } \\
\text { + analg. }\end{array}$ & $\begin{array}{c}\text { Analg. + } \\
\text { distr. }\end{array}$ & 1 & $\begin{array}{c}\text { Pain (WB- } \\
\text { FACES/VAS) } \\
\text { Distress (FLACC) }\end{array}$ & $\begin{array}{l}\text { Opioids + } \\
\text { NSAIDs }\end{array}$ & $\mathrm{Au}$ \\
\hline Morris, 2010* & $\mathrm{CO}$ & $\begin{array}{l}\text { Physical } \\
\text { therapy }\end{array}$ & Burn & Adults & Clin & 11 & 11 & $\begin{array}{l}\text { VR-Distr. } \\
\text { + analg. }\end{array}$ & Analg. & 1 & $\begin{array}{c}\text { Pain (NPRS) } \\
\text { Anxiety (BSPAS) }\end{array}$ & NR & US \\
\hline Schmitt, 2011 & $\mathrm{CO}$ & $\begin{array}{l}\text { Physical } \\
\text { therapy }\end{array}$ & Burn & Mixed & Clin & 54 & 54 & $\begin{array}{l}\text { VR-Distr. } \\
\text { + analg. }\end{array}$ & Analg. & 5 & $\begin{array}{c}\text { Pain (GRS) } \\
\text { Time think (GRS) } \\
\text { Unpleas (GRS) }\end{array}$ & Opioids & US \\
\hline $\begin{array}{l}\text { Schneider, } \\
\text { 2003* }\end{array}$ & $\mathrm{CO}$ & $\begin{array}{c}\text { IV } \\
\text { chemotherap } \\
y\end{array}$ & Cancer Tx. & Adults & Clin & 16 & 16 & VR-Distr. & No Tx. & 1 & $\begin{array}{l}\text { Anxiety (SAI) } \\
\text { Distress (SDS) }\end{array}$ & NR & US \\
\hline Schneider, & $\mathrm{CO}$ & $\begin{array}{c}\text { IV } \\
\text { chemotherap }\end{array}$ & Cancer Tx. & Adults & Clin & 20 & 20 & VR-Distr. & No Tx. & 1 & Anxiety (STAI) & NR & US \\
\hline
\end{tabular}




\begin{tabular}{|c|c|c|c|c|c|c|c|c|c|c|c|c|c|}
\hline Van Twillert* & $\mathrm{CO}$ & Dress Ch & Burn & Child & Clin & 19 & 19 & $\begin{array}{l}\text { VR-Distr } \\
\text { +analg. }\end{array}$ & Analg. & 1 & Pain (VAT) & NR & NL \\
\hline Wolitzky, 2005 & $\mathrm{P}$ & $\begin{array}{c}\text { Port access } \\
\text { placement }\end{array}$ & Cancer Tx. & Child & Clin & 10 & 10 & VR-Distr. & No Tx. & 1 & $\begin{array}{l}\text { Pain (CHEOPS) } \\
\text { Distress (VAS) }\end{array}$ & - & US \\
\hline
\end{tabular}

\section{Note.}

* Trials defined by their authors as pilot or feasibility studies

a) $\mathrm{CO}=$ crossover; $\mathrm{P}=$ parallel

b) Restorative Tx. = dental restorations owing to primary cavities; Dress. Ch. = dressing change; IV Placement $=$ Intravenous $($ IV $)$ placement; IV chemotherapy = Intravenous chemotherapy;

c) Cond, condition; Tx = treatment; TBSA = total body surface area; MRI = magnetic resonance imaging; CT = computed tomography; FDC= first dressing

change; Cancer Tx. = cancer treatment.

d) Age gr = age group; child = children;

e) Clin = participants recruited from clinical settings; comm = participants recruited from community settings;

f) $\mathrm{N}_{\text {rand }} \mathrm{VR}=$ number of participants randomized to the VR intervention;

g) $\mathrm{N}_{\text {rand }}$ control = number of participants randomized to the control intervention;

h) Interv. = Intervention received by the experimental group; VR-Distr.= distraction in VR; VR-RLX= relaxation in VR; VR-Distr. + analg. = Distraction in VR plus analgesics.

i) Control $=$ intervention received by the control group; No Tx. $=$ no treatment received by the control group; Analg. $=$ analgesic only; Analg. + distraction $=$ analgesic + distraction.

j) $\mathrm{N}$ ses = number of sessions;

k) Unpleas = unpleasantness; Time think = time thinking about pain; VAS = visual analog scale; FPS-R = FACES Pain Scale Revised; FLACC = Face, Legs, Activity, Cry, Consolability scale; WB-FACES = Wong-Baker FACES; GRS = Graphic Rating Scale; DFS = Dental Fear Survey; CHEOPS = Children's

Hospital of Eastern Ontario Pain Scale; NRP = Numeric Rating Scale; APPT-WGRS = The Adolescent Pediatric Pain Tool - Word Graphic Rating Scale; NPRS $=$ Numeric Pain Rating Scale; SAI = State-Anxiety Inventory for Adults; SDS = Symptom Distress Scale; VAT = Visual Analog Thermometer;

1) Opioids = morphine and derivate (e.g., codeine, oxycodone); Opioids + NSAIDs $=$ morphine and derivate + Nonsteroidal anti-inflammatory drugs; Local = cream or spray with local administration (e.g., Lidocaine, ELMA cream);

m) Prov, provenience; IR = Iran; DK= Denmark; Au= Australia; ES= Spain; US = United States; CN = China; TR = Turkey; NL= Netherlands. 


\section{Risk of bias in the included studies (Figure 2, Figure S1)}

Most of the included studies were rated as having some concerns or high risk of bias for both parallel and crossover (marked with * in Figure S1) designs. Random sequence generation was rated as some concerns in 13 trials and high risk in 8 trials. For deviations from intended interventions, 13 studies were rated as some concerns, and 8 studies as high risk. All studies were rated as low risk for missing outcome data. All studies used self-report measures. For bias due to selective reporting, based on the trial report and available protocols, 6 trials were rated at high risk and 21 as having some concerns. Only 3 trials were registered (Schmitt et al. 2011; Brown et al. 2014; JahaniShoorab et al. 2015), all retrospectively. Only two trials (Miller et al. 2011; Jeffs et al. 2014) could be rated as low RoB on at least 3 domains.

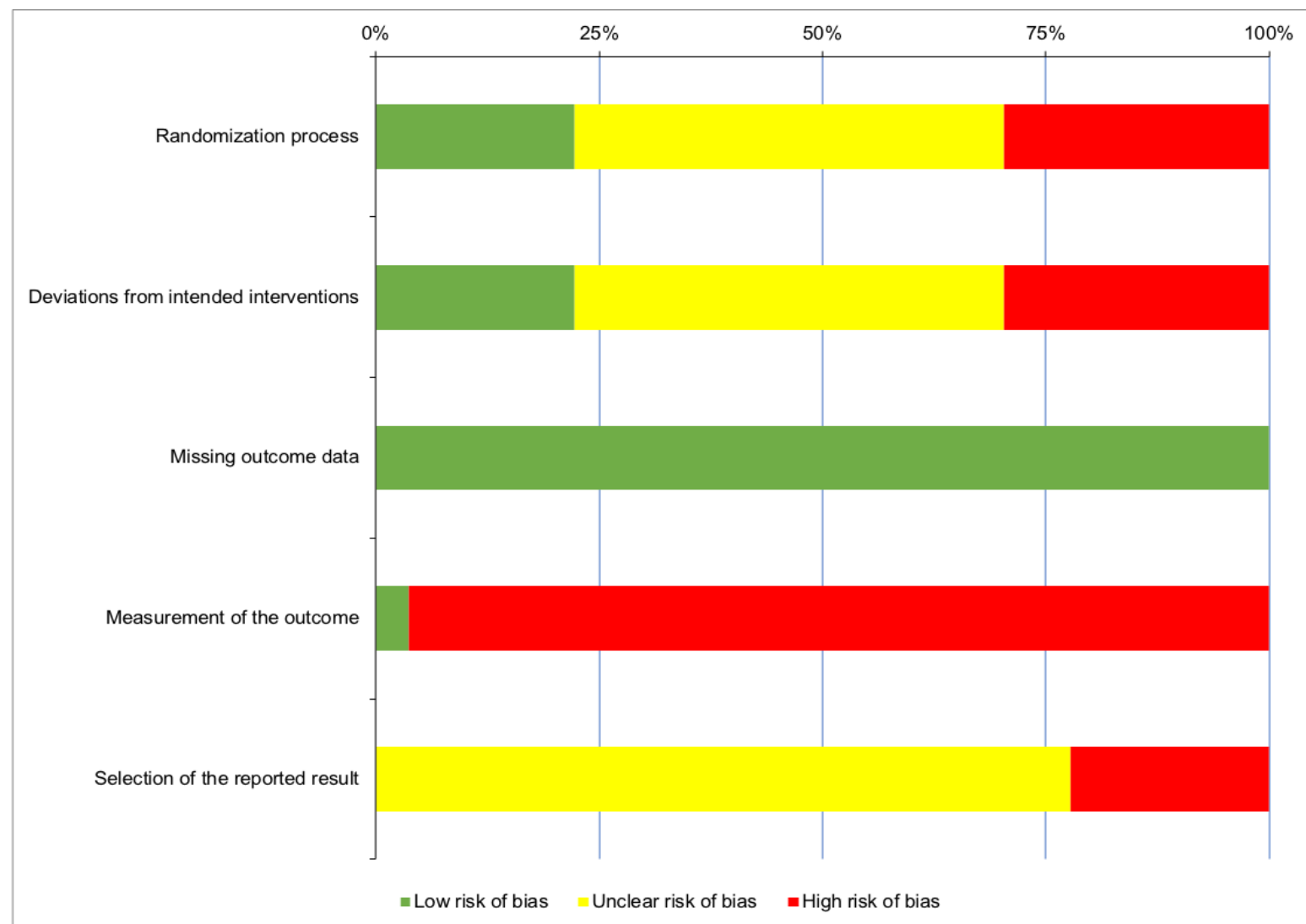

Figure 2. Risk of bias graph: review authors' judgments about each risk of bias item presented as percentages across all included studies. 


\section{VR-based interventions versus TAU (Table 2, Figure 3, 4)}

\section{Pain intensity (primary outcome)}

\section{Real-time}

Nine RCTs (7 parallel) resulted into a Hedges' $g$ of 0.95 (95\% CI 0.32 to 1.57 ), NNT= 2.00, with high heterogeneity $\left(I^{2}=86 \% ; 95 \%\right.$ CI 65 to 96$)$. Sensitivity analyses indicated smaller effects with the Henmi-Copas model, $g=0.77,95 \%$ CI 0.22 to 1.33 , and larger with the Quality Effects model, $g=1.13,95 \%$ CI 0.66 to 1.60 , with heterogeneity remaining high $\left(I^{2}=79 \%\right)$. The effect was reduced in a sensitivity analysis excluding outliers $(n=1), g=0.74$ (95\% CI 0.25 to $1.24), I^{2}=74 \%$ (95\% CI 20 to 94$)$ and when only self-report was considered $(\mathrm{n}=5), g=0.65$ (95\% CI 0.32 to 0.98$), I^{2}=0 \%$ (95\% CI 0 to 82 ). Analyses restricted to children participants, for burns' dressing change, or in parallel designs yielded similar estimations. Six trials with 20 or more participants randomized per arm resulted into a similarly large $g$ of 1.11 (95\% CI 0.07 to 2.15), $I^{2}=90 \%$ (95\% CI 72 to 98). Owing to the high heterogeneity, all PIs, except for selfreported pain, included 0 .

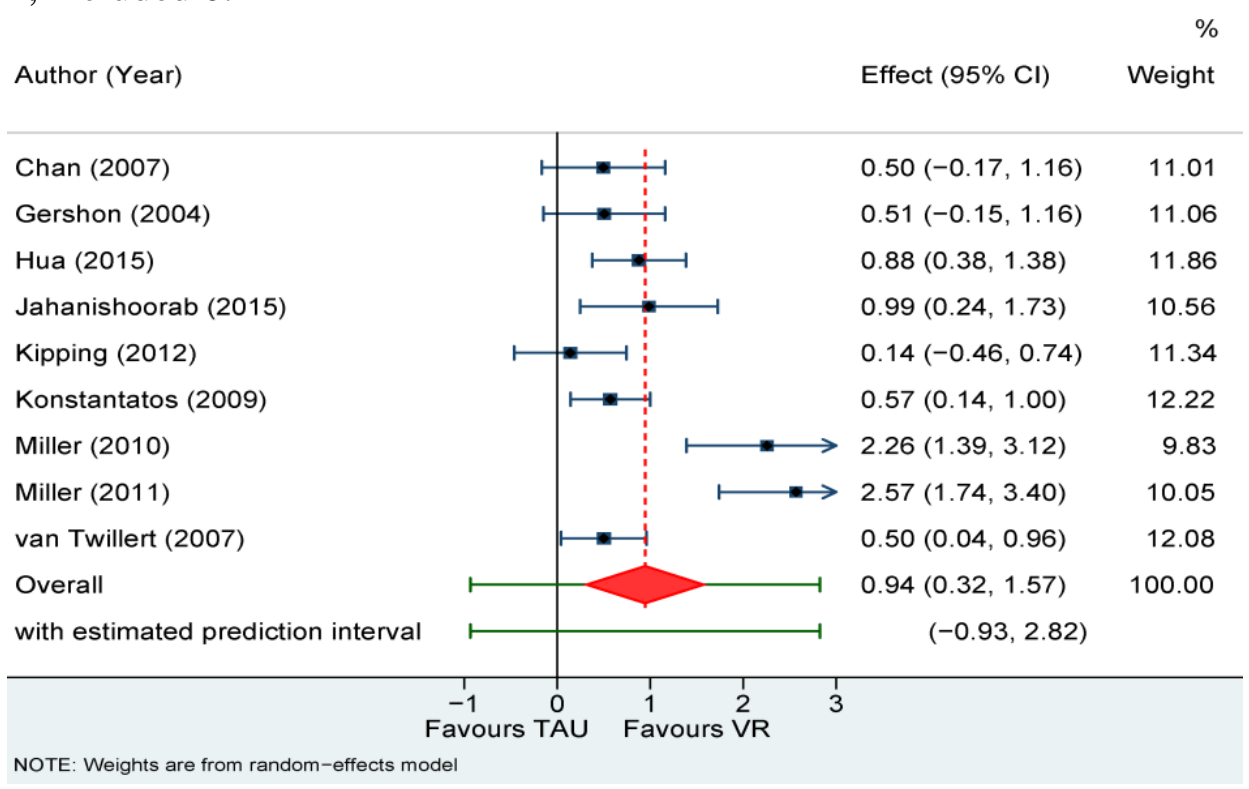

Figure 3. Forest plot of standardized mean differences for pain intensity assessed real-time for VR-based interventions versus treatment as usual. 


\section{Retrospective}

Twenty-two trials resulted into a pooled ES of $\mathrm{g}=0.87$ (95\% CI 0.54 to 1.21$)$, NNT=2.16

with very high heterogeneity, I2 = 89\% (95\% CI 78 to 95). Effects were smaller with the Henmi-

Copas, $\mathrm{g}=0.69,95 \%$ CI 0.36 to 1.01 , and similar with the Quality Effects models, $\mathrm{g}=0.89,95 \%$

CI 0.61 to 1.16 , with heterogeneity remaining high $(\mathrm{I} 2=82 \%)$.

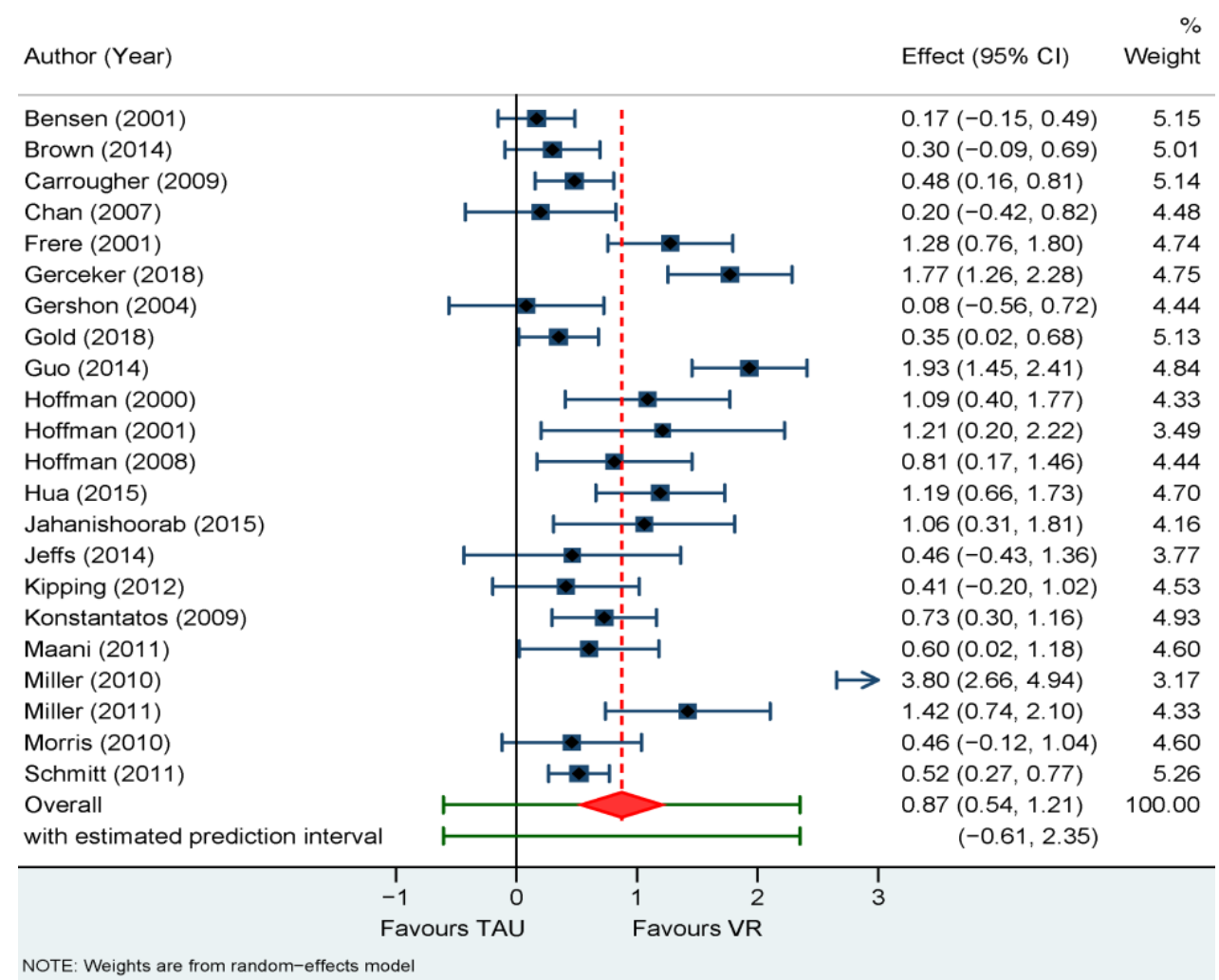

Figure 4. Forest plot of standardized mean differences for pain intensity assessed retrospectively for VR-based interventions versus treatment as usual.

Sensitivity analyses showed decreased ESs with the exclusion of potential outliers $(n=4)$, $\mathrm{g}=0.66(95 \%$ CI 0.46 to 0.85$), \mathrm{I} 2=53 \%(95 \%$ CI 8 to 81$)$, of MMD trials $(\mathrm{n}=3), \mathrm{g}=0.77(95 \%$ CI 0.51 to 1.02$), \mathrm{I} 2=78 \%$ (95\% CI 60 to 90$)$, or of trials with a no intervention control $(\mathrm{n}=4), \mathrm{g}=$ 0.77 (95\% CI 0.41 to 1.14$)$, I2 $=87 \%$ (95\% 68 to 95$)$. Effects were also considerably smaller across crossover trials $(\mathrm{n}=11), \mathrm{g}=0.61(95 \%$ CI 0.34 to 0.88$), \mathrm{I} 2=57 \%$ (95\% CI 1 to 89$)$. Pain 
was self-reported in all but two trials. Analyses restricted to burns dressing change $(n=11), g=1.03$ (95\% CI 0.37 to 1.68$), \mathrm{I} 2=91 \%(95 \% 78$ to 97$)$ or in parallel designs $(\mathrm{n}=11), \mathrm{g}=1.08(95 \% \mathrm{CI}$ 0.46 to 1.70 ), $\mathrm{I} 2=92 \%$ (95\% CI 82 to 98 ) led to slightly higher effects. Effects were similar for children participants $(n=11), g=0.87$ (95\% CI 0.17 to 1.57$), I 2=94 \%$ (95\% 85 to 98$)$, or in trials with at least 20 randomized participants per $\operatorname{arm}(n=14), g=0.97(95 \%$ CI 0.44 to 1.51$), I 2=94 \%$ (95\% CI 87 to 98). All PIs except for the analysis without outliers included 0. 
Table 2. VR-based interventions compared with treatment as usual (TAU)

\begin{tabular}{|c|c|c|c|c|c|c|c|}
\hline & $\mathbf{N}$ & $g^{\mathbf{a}}$ & $95 \% \mathrm{CI}$ & $I^{2}$ & $\begin{array}{c}I^{2} \\
95 \% \mathrm{CI}\end{array}$ & $\begin{array}{c}\text { Predictive } \\
\text { interval } \\
95 \% \mathrm{CI}\end{array}$ & NNT \\
\hline \multicolumn{8}{|c|}{$\begin{array}{l}\text { Sensory component of pain (pain intensity) } \\
\text { measured }\end{array}$} \\
\hline & & & & & \multicolumn{2}{|l|}{65 to 96} & 2.00 \\
\hline Real-time (all assessors) & 9 & 0.95 & 0.32 to 1.57 & 86 & \multicolumn{3}{|c|}{-0.93 to 2.82} \\
\hline Henmi-Copas & 9 & 0.77 & 0.22 to 1.33 & 79 & 65 to 96 & N/A & 2.41 \\
\hline Quality Effects model & 9 & 1.13 & 0.66 to 1.60 & 79 & N/A & N/A & 1.73 \\
\hline Outliers excluded $^{c}$ & 8 & 0.74 & 0.25 to 1.24 & 74 & 20 to 94 & -0.59 to 2.07 & 2.50 \\
\hline Only burns' dressing change & 7 & 1.01 & 0.16 to 1.87 & 90 & 72 to 98 & -1.40 to 3.42 & 1.90 \\
\hline Children participants & 7 & 1.01 & 0.14 to 1.88 & 88 & 70 to 98 & -1.41 to 3.43 & 1.90 \\
\hline Self-report & 5 & 0.65 & 0.32 to 0.98 & 0 & 0 to 82 & 0.27 to 1.03 & 2.82 \\
\hline Other-report & 4 & 1.34 & -0.61 to 3.28 & 91 & 71 to 99 & -4.31 to 6.99 & 1.52 \\
\hline Parallel design $^{\mathrm{d}}$ & 7 & 1.09 & 0.25 to 1.92 & 88 & 67 to 98 & -1.23 to 3.41 & 1.78 \\
\hline $\mathrm{N}>/=20$ randomized/arm & 6 & 1.11 & 0.07 to 2.15 & 90 & 72 to 98 & -1.70 to 3.93 & 1.76 \\
\hline Retrospective (all assessors) & 22 & 0.87 & 0.54 to 1.21 & 89 & 78 to 95 & -0.61 to 2.35 & 2.16 \\
\hline Henmi-Copas & 22 & 0.69 & 0.36 to 1.01 & 82 & 80 to 95 & N/A & 2.67 \\
\hline Quality Effects model & 22 & 0.89 & 0.61 to 1.16 & 82 & & & 2.12 \\
\hline Outliers excluded $^{\mathrm{e}}$ & 18 & 0.66 & 0.46 to 0.85 & 53 & 8 to 81 & 0.06 to 1.25 & 2.78 \\
\hline Excluding MMD & 19 & 0.77 & 0.51 to 1.02 & 78 & 60 to 90 & -0.22 to 1.75 & 2.41 \\
\hline Excluding no tx ctrl ${ }^{\mathrm{f}}$ & 18 & 0.77 & 0.41 to 1.14 & 87 & 68 to 95 & -0.66 to 2.21 & 2.41 \\
\hline Only burns' dressing change & 11 & 1.03 & 0.37 to 1.68 & 91 & 78 to 97 & -1.15 to 3.21 & 1.87 \\
\hline Children participants & 11 & 0.87 & 0.17 to 1.57 & 94 & 85 to 98 & -1.48 to 3.23 & 2.16 \\
\hline \multirow[t]{2}{*}{ Self-report } & 20 & 0.84 & 0.50 to 1.17 & 89 & 79 to 95 & -0.66 to 2.33 & 2.23 \\
\hline & & & & & 1 to 89 & -0.07 to 1.28 & 2.99 \\
\hline Crossover design & 10 & 0.61 & 0.34 to 0.88 & 57 & & & \\
\hline
\end{tabular}




\begin{tabular}{|c|c|c|c|c|c|c|c|}
\hline Parallel design & 12 & 1.08 & 0.46 to 1.70 & 92 & 82 to 98 & -1.06 to 3.22 & 1.80 \\
\hline $\mathrm{N}>/=20$ randomized/arm & 14 & 0.97 & 0.44 to 1.51 & 94 & 87 to 98 & -1.03 to 2.98 & 1.97 \\
\hline Excluding pilots or feasibility studies & 20 & 0.93 & 0.58 to 1.29 & 90 & 79 to 96 & -0.59 to 2.4 & 2.04 \\
\hline \multicolumn{8}{|l|}{$\begin{array}{l}\text { Cognitive component of pain } \\
\text { measured }\end{array}$} \\
\hline Retrospective & 8 & 0.82 & 0.39 to 1.26 & 75 & 24 to 95 & -0.29 to 1.94 & 2.28 \\
\hline \multicolumn{8}{|l|}{ Affective component of pain measured } \\
\hline Real-time & 5 & 0.94 & 0.33 to 1.56 & 51 & 0 to 94 & -0.37 to 2.25 & 2.02 \\
\hline Retrospective & 14 & 0.55 & 0.34 to 0.77 & 58 & 4 to 86 & -0.08 to 1.19 & 3.30 \\
\hline
\end{tabular}

\section{Note.}

$\mathrm{N}=$ number of studies; NNT= numbers needed to treat; Child $=$ children; Dress $\mathrm{Ch}=$ dressing change; Phys $=$ Physical; $\mathrm{Tx}=\mathrm{Therapy} ; \mathrm{Ctrl}=\mathrm{control} ; \mathrm{Conc}=$ concomitent; Analg = analgesic; Distr= distraction; VR = Virtual Reality; HMD = Head-Mounted Display; VG = Video Glasses; MMD = Multi-modal device, $\mathrm{RoB}=$ risk of bias, $\mathrm{N} / \mathrm{A}=$ not available

a All results are reported with Hedges' $g$, using a random effects model, positive effect indicates superiority of the experimental group over control group (significant results are marked with italic).

${ }^{c}$ Miller, 2011

${ }^{d}$ The two crossover studies were both identified by the authors as pilot or feasibility studies

${ }^{\mathrm{e}}$ Bensen, 2001; Gerceker, 2018; Guo, 2014, Miller, 2010

${ }^{\mathrm{f}}$ Excluding trials with a no treatment control arm 


\section{Affective and cognitive components of pain (secondary outcome)}

Five studies assessed the affective component of pain real-time, $g=0.94$ (95\% CI 0.33 to 1.56), NTT $=2.02, I^{2}=51 \%(95 \%, 0$ to 94$)$ and 14 trials retrospectively, $g=0.55$ (95\% CI 0.34 to

0.77), $\mathrm{NNT}=3.30, I^{2}=58 \%(95 \% \mathrm{CI} 4$ to 86$)$. The cognitive component was assessed only retrospectively in eight trials, $g=0.82(95 \% \mathrm{CI} 0.39$ to 1.26$), \mathrm{NTT}=2.28, I^{2}=75 \%(95 \% \mathrm{CI} 24$ to 95).

\section{VR-based interventions versus active comparators}

Two studies assessed pain intensity real-time and four studies retrospectively, $g=0.69$ (95\% CI -0.58 to 1.97$), I^{2}=83 \%$ (95\% CI 43 to 99 ), PI -2.86 to 4.25 . The affective component was assessed in 2 studies.

\section{Adverse effects (Table S1)}

Twelve studies evaluated potential nausea or simulator sickness associated with VR interventions. In one, $15 \%$ of the participants reported nausea, and in another $5.2 \%$ reported nausea and $8 \%$ simulator sickness. In the remaining trials, none or under $5 \%$ of participants reported nausea.

\section{Subgroup analysis}

We only conducted planned subgroup analyses for VR-based interventions versus TAU for pain intensity assessed retrospectively. The two characteristics planned were correlated (Cramér's $V=-0.57$ ), therefore analyses were only conducted with analgesic use. Differences 
between studies using concomitant analgesic $(n=16, g=0.78,95 \%$ CI 0.37 to 1.19$)$ versus those that did not $(\mathrm{n}=6 \mathrm{~g}=1.09,95 \%$ CI 0.33 to 1.86$)$ were not significant, $\mathrm{F}(1,20)=0.86, p=0.36$.

\section{Small study effects (Figure S2)}

These were gauged for pain intensity assessed retrospectively ( 22 trials). The funnel plot appeared asymmetrical (Figure S2 A), and visualization with contour enhanced funnel plot (Figure S2 B) suggested that most studies were significant at the conventional threshold of $p<.05$. Egger's test was significant (intercept $=3.09,95 \%$ CI 0.50 to $5.67, p=0.021$ ).

\section{Discussion}

In a meta-analysis of twenty-seven randomized trials, VR-based distraction interventions for procedural pain demonstrated reductions in pain intensity, assessed either real-time or retrospectively, compared to treatment as usual. Though effects appeared generally large, they were associated by high heterogeneity, with all predictive intervals including zero. Effectively, this implies that the effects of $95 \%$ of future similar trials fluctuate across a wide range of effects, both favorable and not to VR-based interventions. Across several sensitivity analyses, involving both alternative statistical models (i.e., robust to publication bias, considering study quality), and restricted to the largest, clinically relevant and more homogenous categories (e.g., children participants, burn dressing change procedures), heterogeneity remained high and effect estimates largely similar. VR-based interventions were also effective for the affective and cognitive components of pain, assessed retrospectively, though the number of trials was more limited. Only four studies contrasted VR-based interventions with active comparators with a non-significant but large effect. Adverse effects were reported in a minority of participants and mostly consisted of nausea and simulator sickness. 
Despite these seemingly promising effects, serious methodological and reporting issues across the entire evidence base preclude any inferences regarding clinical effectiveness. First, trial risk of bias was rated as high or raising some concerns for most of the included trials for randomization, deviations from the intended intervention and selective reporting. In most instances, ratings were motivated by the absence of essential information for the assessment of these domains. Only three trials were registered retrospectively, and just one had a published protocol (Brown et al. 2014). Crossover trials in particular were missing essential, often basic, descriptive information, such as the comparative baseline characteristics of participants randomized to receive the VR-intervention first (i.e., $\mathrm{AB}$ sequence) or last (i.e., BA sequence), reported in none of the trials. As the VR-based intervention involved specialized equipment, it was generally impossible to blind participants and the personnel administering it. Also, owing to the outcome (pain) or the assessment timepoint (real-time), all studies relied on self-report measures or used unblinded observers (i.e., parent, nurse, researchers).

The gaps in reporting also translated in the frequent absence of data necessary for effect estimation, particularly for crossover trials, where initially only half included usable data. Attempts to contact the authors in order to recover necessary data, even though repeated, were generally unsuccessful, leaving out almost a third of eligible trials from the meta-analysis. Missing information most likely affected the precision of the effect estimates, exposing our metaanalysis to the risk of selective reporting, and hampered a more meaningful exploration of moderator effects.

Other important caveats relate to heterogeneity around effect estimates being generally high and even extremely high, or with large confidence intervals (Jackson \& Bowden 2016) across most analyses, and generally not diminished in sensitivity analyses. High heterogeneity 
impacts the precision of the effect estimates, and raises questions about their reliability (Ioannidis et al. 2007). Several analyses relied on a small number of studies and most included trials had small sample sizes. We tried to counteract these limitations by choosing a statistical approach resilient to bias in meta-analysis of small or few studies (Röver et al. 2015; Veroniki et al. 2016), and by conducting several robustness analyses. There was also evidence of small study effects, and possible publication bias, with the pooled standardized mean differences reduced by 0.20 on average when the Henmi-Copas, a statistical model robust to publication bias was employed.

Our findings diverge from and extend those of a recent meta-analysis (Chan et al. 2018), reporting a moderate effect of VR interventions for self-reported ratings of worst pain. We used a larger array of pain intensity outcomes and distinguished between pain reported real-time and retrospectively, as well as between self- and other-report. Though generally larger, our estimates were similar in several sensitivity analyses. Most of the included studies focused on burns, where VR-based interventions appeared particularly effective for reducing pain intensity associated with dressing change. Scheffler and colleagues (2017) also found a large effect for VR-based distraction for burn wound care in adults, though they combined pain intensity and other pain components and did not distinguish between dressing change and physical therapy. A very limited number of trials assessed other types of procedural pain, such as dental or needle related (e.g., IV placement). About half of the trials included in the meta-analysis involved children participants, resulting into estimates and heterogeneity nearly identical as the overall ones. Sensitivity analyses excluding studies using the MMD device considerably reduced the pooled effect size. Use of concomitant analgesic was not a moderator, though the number of studies in the "absent" subgroup was disproportionately small. The dosage of analgesic received was usually not 
reported. Only one study (Carrougher et al. 2009) examined whether VR-based interventions reduced concomitant analgesic use, with non-significant results.

\section{Conclusions}

Interpreting these results is a glass half-full/half-empty conundrum. The setting is challenging, with large trials absent and difficult to conduct. For several indications, such as burns, particularly with children, recruiting a reasonably large number of participants to be randomized is difficult. Moreover, VR-based interventions were, until recently, difficult to scale. Not coincidentally, half of the trials we included were cross-over. Procedural pain is an unavoidable side-effect in settings such as burn care, compelling medical staff, patients and caregivers to try to alleviate it by any intervention that appears safe. Distraction intervention are generally effective (Scheffler et al. 2017) for adults, but less so for children and adolescents (Birnie et al. 2014). A new technology like VR, purported to enhance the effectiveness of "regular" distraction, will likely be embraced. Moreover, a cost analysis simulation estimated a using adjuvant VR therapy for pain management in hospitalized patients would reduce costs by \$5.4/patient (95\% CI \$11 to \$156) compared with TAU (Delshad et al. 2018). Hence, our metaanalysis provides reassurance VR-based distraction interventions appear safe and with some benefits in reducing procedural pain.

Conversely, our results were based on small trials, at risk of bias in their design, implementation and reporting. The overall quality of evidence for VR-based interventions is poor, barring any meaningful implications for clinical practice. The patchy and often non-transparent reporting of trials hinders progress in the field, by stymieing larger scale replication and accurate assessment of effects. If anything, our findings amount to establishing proof-of-concept. 
Conclusions about clinical effectiveness and any potential for real-world implementation at a wider scale should be based on larger, prospectively registered and transparently reported Phase 3 trials. These could extend to other medical conditions than burns, such as cancer treatment or post-surgical pain, focus more on clinically relevant assessment points for pain, such as real-time and include relevant clinical outcomes like changes in analgesics dose and type. 


\section{List of included tables (main)}

Table 1. Characteristics of included studies

Table 2. VR-based interventions compared with treatment as usual (TAU)

\section{List of included figures (main)}

Figure 1. PRISMA flow-diagram of the study selection process.

Figure 2. Risk of bias graph: review authors' judgments about each risk of bias item presented as percentages across all included studies.

Figure 3. Forest plot of standardized mean differences for pain intensity assessed real-time for VR-based interventions versus treatment as usual.

Figure 4. Forest plot of standardized mean differences for pain intensity assessed retrospectively for VR-based interventions versus treatment as usual. 


\section{Data sharing statement}

All extracted data used for effect size calculation and the STATA code for conducting metaanalysis (with the exception of study level effect size calculations done in Comprehensive Metaanalysis) are available on the Open Science Framework (DOI 10.17605/OSF.IO/J2QCF)

\section{Financial support}

Raluca Georgescu, Liviu A. Fodor and Ioana A. Cristea were supported by a grant from the Romanian Ministery of Research and Innovation, CNCS - UEFISCDI (project number PNIII-P1-1.1-TE-2016-1054). The funder had no role in the design of the study, collection, analysis and interpretation of data, and the decision to approve publication.

\section{Conflict of interest}

All authors have completed the ICMJE uniform disclosure form at http://www.icmje.org/coi_disclosure.pdf (available upon request from the corresponding author) and have nothing to disclose.

\section{Author contributions}

Author contributions (with author initials): Conceptualization (IAC); Methodology (RG, LAF, IAC); Data curation (RG, LAF); Formal analysis (RG, LAF); Supervision (IAC, AD); Writing- original draft (RG, LAF); Writing- reviews \& editing (IAC, AD). 


\section{References}

Barr J, Fraser GL, Puntillo K, Ely EW, Gélinas C, Dasta JF, Davidson JE, Devlin JW, Kress JP, Joffe AM, Coursin DB, Herr DL, Tung A, Robinson BRH, Fontaine DK, Ramsay MA, Riker RR, Sessler CN, Pun B, Skrobik Y, Jaeschke R, American College of Critical Care Medicine (2013). Clinical practice guidelines for the management of pain, agitation, and delirium in adult patients in the intensive care unit. Critical Care Medicine 41, 263-306.

Bentsen B, Svensson P, Wenzel A (2001). Evaluation of effect of 3D video glasses on perceived pain and unpleasantness induced by restorative dental treatment. European Journal of Pain (London, England) 5, 373-378.

Birnie KA, Noel M, Parker JA, Chambers CT, Uman LS, Kisely SR, McGrath PJ (2014). Systematic Review and Meta-Analysis of Distraction and Hypnosis for Needle-Related Pain and Distress in Children and Adolescents. Journal of Pediatric Psychology 39, 783-808.

Borenstein M, Hedges LV, Higgins JPT, Rothstein HR (2009). Introduction to Meta-Analysis. 1 st edn Statistics in Practice. Wiley.

Breivik H, Eisenberg E, O'Brien T, OPENMinds (2013). The individual and societal burden of chronic pain in Europe: the case for strategic prioritisation and action to improve knowledge and availability of appropriate care. BMC public health 13, 1229.

Brooks FP (1999). What's Real About Virtual Reality? IEEE Computer Graphics and Applications 19, 16-27.

Brown NJ, Kimble RM, Rodger S, Ware RS, Cuttle L (2014). Play and heal: Randomized controlled trial of DittoTM intervention efficacy on improving re-epithelialization in pediatric burns. burns 40 (2014) 204-213

Carrougher GJ, Hoffman HG, Nakamura D, Lezotte D, Soltani M, Leahy L, Engrav LH, Patterson DR (2009). The Effect of Virtual Reality on Pain and Range of Motion in Adults With Burn Injuries. Journal of burn care \& research : official publication of the American Burn Association 30, 785-791.

Chan E, Foster S, Sambell R, Leong P (2018). Clinical efficacy of virtual reality for acute procedural pain management: A systematic review and meta-analysis. PloS One 13, e0200987.

Chan EY, Blyth FM, Nairn L, Fransen M (2013). Acute postoperative pain following hospital discharge after total knee arthroplasty. Osteoarthritis and Cartilage 21, 1257-1263.

Chan EA, Chung JW, Wong TK, Lien AS and Yang JY (2007) Application of a virtual reality prototype for pain relief of pediatric burn in Taiwan. Journal of Clinical Nursing 16, 786-793.

da Costa RSM, Ribeiro S do N, Cabral ED (2012). Determinants of painful experience during dental treatment. Revista Dor 13, 365-370. 
Delshad SD, Almario CV, Fuller G, Luong D, Spiegel BMR (2018). Economic analysis of implementing virtual reality therapy for pain among hospitalized patients. npj Digital Medicine 1, 22.

Doi SAR, Barendregt JJ, Khan S, Thalib L, Williams GM (2015). Advances in the metaanalysis of heterogeneous clinical trials II: The quality effects model. Contemporary Clinical Trials 45, 123-129.

Egger M, Davey Smith G, Schneider M, Minder C (1997). Bias in meta-analysis detected by a simple, graphical test. BMJ (Clinical research ed.) 315, 629-634.

Elbourne DR, Altman DG, Higgins JPT, Curtin F, Worthington HV, Vail A (2002). Metaanalyses involving cross-over trials: methodological issues. International Journal of Epidemiology 31, 140-149.

Fisher D (2019). ADMETAN: Stata module to provide comprehensive meta-analysis. Boston College Department of Economics.

Fisher DJ (2015). Two-stage Individual Participant Data Meta-analysis and Generalized Forest Plots. The Stata Journal 15, 369-396.

Frere CL, Crout R, Yorty J and McNeil DW (2001) Effects of audiovisual distraction during dental prophylaxis. Journal of the American Dental Association (1939) 132, 1031-1038

Gallace A, Ngo MK, Sulaitis J, Spence C (2012). Multisensory Presence in Virtual Reality: Possibilities \& Limitations. Multiple Sensorial Media Advances and Applications: New Developments in MulSeMedia, 1-38.

Gerçeker GÖ, Binay Ş, Bilsin E, Kahraman A, Yılmaz HB (2018). Effects of Virtual Reality and External Cold and Vibration on Pain in 7- to 12-Year-Old Children During Phlebotomy: A Randomized Controlled Trial. Journal of Perianesthesia Nursing: Official Journal of the American Society of PeriAnesthesia Nurses

Gershon J, Zimand E, Pickering M, Rothbaum BO, Hodges L (2004). A pilot and feasibility study of virtual reality as a distraction for children with cancer. Journal of the American Academy of Child and Adolescent Psychiatry 43, 1243-1249.

Gold JI, Belmont KA, Thomas DA (2007). The Neurobiology of Virtual Reality Pain Attenuation. CyberPsychology \& Behavior 10, 536-544.

Gold JI, Kim SH, Kant AJ, Joseph MH and Rizzo AS (2006) Effectiveness of virtual reality for pediatric pain distraction during i.v. placement. Cyberpsychology \& Behavior: The Impact of the Internet, Multimedia and Virtual Reality on Behavior and Society 9, 207-212.

Gold JI, Mahrer NE (2018). Is Virtual Reality Ready for Prime Time in the Medical Space? A Randomized Control Trial of Pediatric Virtual Reality for Acute Procedural Pain Management. Journal of Pediatric Psychology 43, 266-275. 
Guo C, Deng H and Yang J (2015) Effect of virtual reality distraction on pain among patients with hand injury undergoing dressing change. Journal of Clinical Nursing 24, 115-120

Harbord RM, Harris RJ, Sterne JAC (2009). Updated Tests for Small-study Effects in Metaanalyses. The Stata Journal 9, 197-210.

Hartung J, Knapp G (2001). On tests of the overall treatment effect in meta-analysis with normally distributed responses. Statistics in Medicine 20, 1771-1782.

Hedges LV, Olkin I (1985). Statistical methods for meta-analysis. vol San Diego, CA: Academic Press

Henmi M, Copas JB (2010). Confidence intervals for random effects meta-analysis and robustness to publication bias. Statistics in Medicine 29, 2969-2983.

Higgins I, Green S (2011). Cochrane Handbook for Systematic Reviews of Interventions Version 5.1.0 [updated March 2011].

Higgins JPT, Sterne J, Savović J, Page M, Hróbjartsson, Boutron I, Reeves B, Eldridge S (2016). A revised tool for assessing risk of bias in randomized trials. Cochrane Methods Cochrane Database of Systematic Reviews 2016

Higgins JPT, Thompson SG, Deeks JJ, Altman DG (2003). Measuring inconsistency in metaanalyses. BMJ (Clinical research ed.) 327, 557-560.

Higgins JPT, Thompson SG, Spiegelhalter DJ (2009). A re-evaluation of random-effects metaanalysis. Journal of the Royal Statistical Society. Series A, (Statistics in Society) 172, 137-159.

Hoffman HG, Patterson DR and Carrougher GJ (2000) Use of virtual reality for adjunctive treatment of adult burn pain during physical therapy: a controlled study. The Clinical Journal of Pain 16, 244-250.

Hoffman HG, Patterson DR, Carrougher GJ and Sharar SR (2001) Effectiveness of virtual reality-based pain control with multiple treatments. The Clinical Journal of Pain 17, 229-235.

Hoffman HG, Patterson DR, Seibel E, Soltani M, Jewett-Leahy L and Sharar SR (2008) Virtual reality pain control during burn wound debridement in the hydrotank. The Clinical Journal of Pain 24, 299-304.

Hua Y, Qiu R, Yao W-Y, Zhang Q and Chen X-L (2015) The Effect of Virtual Reality Distraction on Pain Relief During Dressing Changes in Children with Chronic Wounds on Lower Limbs. Pain Management Nursing: Official Journal of the American Society of Pain Management Nurses 16, 685-691

Ioannidis JPA, Patsopoulos NA, Evangelou E (2007). Uncertainty in heterogeneity estimates in meta-analyses. BMJ (Clinical research ed.) 335, 914-916. 
Jackson D, Bowden J (2016). Confidence intervals for the between-study variance in randomeffects meta-analysis using generalised heterogeneity statistics: should we use unequal tails? BMC Medical Research Methodology 16

JahaniShoorab N, Ebrahimzadeh Zagami S, Nahvi A, Mazluom SR, Golmakani N, Talebi M, Pabarja F (2015). The Effect of Virtual Reality on Pain in Primiparity Women during Episiotomy Repair: A Randomize Clinical Trial. Iranian Journal of Medical Sciences 40, 219 224.

Jeffs D, Dorman D, Brown S, Files A, Graves T, Kirk E, Meredith-Neve S, Sanders J, White B, Swearingen CJ (2014). Effect of virtual reality on adolescent pain during burn wound care. Journal of Burn Care \& Research: Official Publication of the American Burn Association 35, 395-408.

Kipping B, Rodger S, Miller K and Kimble RM (2012) Virtual reality for acute pain reduction in adolescents undergoing burn wound care: a prospective randomized controlled trial. Burns: Journal of the International Society for Burn Injuries 38, 650-657

Kleiber C, Harper DC (1999). Effects of distraction on children's pain and distress during medical procedures: a meta-analysis. Nursing Research, 48, 44-49.

Konstantatos AH, Angliss M, Costello V, Cleland H and Stafrace S (2009) Predicting the effectiveness of virtual reality relaxation on pain and anxiety when added to PCA morphine in patients having burns dressings changes. Burns: Journal of the International Society for Burn Injuries 35, 491-499.

Kraemer HC, Kupfer DJ (2006). Size of Treatment Effects and Their Importance to Clinical Research and Practice. Biological Psychiatry 59, 990-996.

Laupacis A, Sackett DL, Roberts RS (1988). An assessment of clinically useful measures of the consequences of treatment. The New England Journal of Medicine 318, 1728-1733.

Li L, Yu F, Shi D, Shi J, Tian Z, Yang J, Wang X, Jiang Q (2017). Application of virtual reality technology in clinical medicine. American Journal of Translational Research 9, 38673880 .

Maani CV, Hoffman HG, Morrow M, Maiers A, Gaylord K, McGhee LL, DeSocio PA (2011). Virtual reality pain control during burn wound debridement of combat-related burn injuries using robot-like arm mounted VR goggles. The Journal of Trauma 71, S125-130.

Malloy KM, Milling LS (2010). The effectiveness of virtual reality distraction for pain reduction: a systematic review. Clinical Psychology Review 30, 1011-1018.

Miller K, Rodger S, Bucolo S, Greer R and Kimble RM (2010) Multi-modal distraction. Using technology to combat pain in young children with burn injuries. Burns: Journal of the International Society for Burn Injuries 36, 647-658 
Miller K, Rodger S, Kipping B, Kimble RM (2011). A novel technology approach to pain management in children with burns: A prospective randomized controlled trial. Burns: Journal of the International Society for Burn Injuries 37, 395-405.

Moher D, Liberati A, Tetzlaff J, Altman DG, PRISMA Group (2009). Preferred reporting items for systematic reviews and meta-analyses: the PRISMA statement. PLoS medicine 6, e1000097.

Morris LD, Louw QA and Crous LC (2010) Feasibility and potential effect of a low-cost virtual reality system on reducing pain and anxiety in adult burn injury patients during physiotherapy in a developing country. Burns: Journal of the International Society for Burn Injuries 36, 659-664.

Norman AT, Judkins KC (2004). Pain in the patient with burns. Continuing Education in Anaesthesia Critical Care \& Pain 4, 57-61.

Pak JG, White SN (2011). Pain Prevalence and Severity before, during, and after Root Canal Treatment: A Systematic Review. Journal of Endodontics 37, 429-438.

Palmer TM, Sutton AJ, Peters JL, Moreno SG (2008). Contour-Enhanced Funnel Plots for Meta-Analysis. The Stata Journal: Promoting communications on statistics and Stata 8, 242-254.

Paule RC, Mandel J (1982). Consensus Values and Weighting Factors. Journal of Research of the National Bureau of Standards 87, 377.

Peters JL, Sutton AJ, Jones DR, Abrams KR, Rushton L (2008). Contour-enhanced metaanalysis funnel plots help distinguish publication bias from other causes of asymmetry. Journal of Clinical Epidemiology 61, 991-996.

Piskorz J, Czub M (2014). Distraction of attention with the use of virtual reality. Influence of the level of game complexity on the level of experienced pain. Polish Psychological Bulletin 45, 480487.

Röver C, Knapp G, Friede T (2015). Hartung-Knapp-Sidik-Jonkman approach and its modification for random-effects meta-analysis with few studies. BMC Medical Research Methodology 15, 99.

Scheffler M, Koranyi S, Meissner W, Strauß B, Rosendahl J (2017). Efficacy of nonpharmacological interventions for procedural pain relief in adults undergoing burn wound care: A systematic review and meta-analysis of randomized controlled trials. Burns: Journal of the International Society for Burn Injuries

Schmitt YS, Hoffman HG, Blough DK, Patterson DR, Jensen MP, Soltani M, Carrougher GJ, Nakamura D, Sharar SR (2011). A randomized, controlled trial of immersive virtual reality analgesia, during physical therapy for pediatric burns. Burns: Journal of the International Society for Burn Injuries 37, 61-68. 
Schneider SM, Ellis M, Coombs WT, Shonkwiler EL and Folsom LC (2003) Virtual reality intervention for older women with breast cancer. Cyberpsychology \& Behavior: The Impact of the Internet, Multimedia and Virtual Reality on Behavior and Society 6, 301-307.

Schneider SM, Prince-Paul M, Allen MJ, Silverman P and Talaba D (2004) Virtual reality as a distraction intervention for women receiving chemotherapy. Oncology Nursing Forum 31, 8188

Seers K, Carroll D (1998). Relaxation techniques for acute pain management: a systematic review. Journal of Advanced Nursing 27, 466-475.

Sidik K, Jonkman JN (2002). A simple confidence interval for meta-analysis. Statistics in Medicine 21, 3153-3159.

Sommer M, Rijke JM de, Kleef M van, Kessels AGH, Peters ML, Geurts JWJM, Gramke H-F, Marcus M a. E (2008). The prevalence of postoperative pain in a sample of 1490 surgical inpatients. European Journal of Anaesthesiology 25, 267-274.

Torrati FG, Rossi LA, Ferreira E, Dalri MC, de Carvalho EC, dos Santos Barbeira CB (2000). Analysis of cost of dressings in the care of burn patients. Burns: Journal of the International Society for Burn Injuries 26, 289-293.

Tsirigotou S (1993). Acute and chronic pain resulting from burn injuries. Ann. Medit. Burns Clu, 6,1 .

Turk DC, Okifuji A (2002). Psychological factors in chronic pain: evolution and revolution. Journal of Consulting and Clinical Psychology 70, 678-690.

Uman LS, Chambers CT, McGrath PJ, Kisely S (2008). A Systematic Review of Randomized Controlled Trials Examining Psychological Interventions for Needle-related Procedural Pain and Distress in Children and Adolescents: An Abbreviated Cochrane Review. Journal of Pediatric Psychology 33, 842-854.

van den Beuken-van Everdingen MHJ, Hochstenbach LMJ, Joosten EAJ, Tjan-Heijnen VCG, Janssen DJA (2016). Update on Prevalence of Pain in Patients With Cancer: Systematic Review and Meta-Analysis. Journal of Pain and Symptom Management 51, 1070-1090.e9.

Verhoeven K, Crombez G, Eccleston C, Van Ryckeghem DML, Morley S, Van Damme S (2010). The role of motivation in distracting attention away from pain: an experimental study. Pain 149, 229-234.

Verhoeven K, Dick B, Eccleston C, Goubert L, Crombez G (2014). The role of executive functioning in children's attentional pain control: An experimental analysis. PAIN® 155, 413421. 
Veroniki AA, Jackson D, Viechtbauer W, Bender R, Bowden J, Knapp G, Kuss O, Higgins JP, Langan D, Salanti G (2016). Methods to estimate the between-study variance and its uncertainty in meta-analysis. Research Synthesis Methods 7, 55-79.

Viechtbauer W (2007). Confidence intervals for the amount of heterogeneity in meta-analysis. Statistics in Medicine 26, 37-52.

Walker MR, Kallingal GJS, Musser JE, Folen R, Stetz MC, Clark JY (2014). Treatment efficacy of virtual reality distraction in the reduction of pain and anxiety during cystoscopy. Military Medicine 179, 891-896.

Wint SS, Eshelman D, Steele J, Guzzetta CE (2002). Effects of Distraction Using Virtual Reality Glasses During Lumbar Punctures in Adolescents With Cancer. Oncology Nursing Forum 29, E8-E15.

Wolitzky K, Fivush R, Zimand E, Hodges L and Rothbaum BO (2005) Effectiveness of virtual reality distraction during a painful medical procedure in pediatric oncology patients. Psychology \& Health 20, 817-824 